\title{
Um Ensaio sobre os Programas de Pesquisa Lakatosianos e a Metodologia da Economia Neoclássica: Contribuições e Críticas
}

\section{An Essay on Lakatosian Scientific Research Programs and Neoclassical Economics Methodology: Contributions and Critics}

\author{
Ricardo Agostini Martini*
}

\begin{abstract}
Resumo: A obra de Imre Lakatos procurou proporcionar um refinamento à abordagem falsificacionista popperiana, que o inspirou, mediante a incorporação de conceitos desenvolvidos por Thomas Kuhn, mas sem negar algumas das hipóteses clássicas do falsificacionismo, como os critérios demarcacionistas e a ênfase na investigação e nos testes empíricos. As principais ideias do autor consistem na noção de programa de pesquisa como uma estrutura dotada de um núcleo irredutível de hipóteses básicas das teorias levantadas, protegido por um cinturão protetor de hipóteses auxiliares e por heurísticas positivas e negativas que guiam o processo de investigação científica. O pensamento de Lakatos foi discutido na metodologia da economia por parecer defender boa parte do trabalho teórico e empírico dessa profissão. Contudo, apesar de um entusiasmo inicial, a aplicação dos programas de pesquisa lakatosianos na ciência econômica passaram a ser criticados pela bibliografia em geral a partir dos anos 1980 . Parte dessas críticas dirigiu-se diretamente a Lakatos; outra se dirigiu ao pensamento falsificacionista; e outras atacaram os próprios critérios demarcacionistas da metodologia científica. O presente estudo busca realizar uma revisão bibliográfica sobre os principais pontos destacados nesse debate.
\end{abstract}

Palavras-chave: Metodologia da economia. Imre Lakatos. Falsificacionismo. História do pensamento econômico.

\begin{abstract}
The work of Imre Lakatos intended to present a refinement to popperian falsificationism approach that inspired him, by incorporating concepts developed by Thomas Kuhn, but without denying some of the classical hypothesis of falsificationism, as demarcationist rules and emphasis on investigation and on empirical tests. The author's main ideas consists in the notion of a research program like a structure endowed with a hardcore of basic hypothesis about raised theories, and protected by a belt of auxiliary hypothesis and by positives and negatives heuristics that guide the scientific investigation process. Lakatos thought was discussed in methodology of economics because it appeared to explain the main work, theoretical and empirical, of this profession. However, despite an initial enthusiasm, the application of lakatosian research programs in economics was criticized as of the eighties. Some of these critics addressed

Economista do Banco Nacional de Desenvolvimento Econômico e Social (BNDES). Mestre em Economia pelo Centro de Desenvolvimento e Planejamento Regional da Universidade Federal de Minas Gerais (Cedeplar/UFMG). E-mail: ricardoamartini@gmail.com O autor agradece as contribuições do Prof. Dr. Hugo Eduardo Araújo da Gama Cerqueira (Cedeplar/UFMG) para a elaboração do presente trabalho.
\end{abstract}


directly to Lakatos; some addressed to falsificationist thought; and some critics attacked the demarcationist rules of scientific methodology. The present paper intends to do a bibliographical revision about the main ideas outstanding in this discussion.

Keywords: Economic methodology. Imre Lakatos. Falsificationism. History of economic thought.

JEL Classification: B31; B41.

\section{Introdução}

Imre Lakatos foi um dos principais nomes da filosofia da ciência no século XX. Basicamente, sua obra procurou proporcionar um refinamento à abordagem falsificacionista popperiana, que o inspirou mediante a incorporação de conceitos desenvolvidos por Thomas Kuhn (1982), mas sem negar algumas das hipóteses clássicas do falsificacionismo, como as de critérios demarcacionistas e a da ênfase na investigação e nos testes empíricos. Para a ciência econômica, o pensamento de Lakatos é interessante por parecer defender boa parte do trabalho teórico e empírico da sua profissão.

Em resumo, Imre Lakatos tentou resgatar as ideias de Popper (1996) frente às críticas de Kuhn (1982). ${ }^{1}$ Lakatos aceitou o falsificacionismo, mas propôs que as teorias científicas apresentam "núcleos" de hipóteses que não podem ser testadas e refutadas por testes empíricos. Por outro lado, defendeu que o processo no qual os cientistas escolhem seus paradigmas (que o autor denominou de "programas de pesquisa”) não é um fenômeno de psicologia social, mas sim uma escolha racional, de modo que os indivíduos procuram pesquisar utilizando a metodologia que lhes dê a maior possibilidade de sucesso. Segundo Lakatos (1978, p. 19), “[...] se tivermos dois programas de investigação rivais, um deles progressivo e o outro degenerativo, os cientistas tendem a aderir ao programa progressivo. Esta é a base racional das revoluções científicas".

Na segunda parte do artigo, são explicados os conceitos e ideias fundamentais da metodologia da ciência em Imre Lakatos. A noção de programa de pesquisa como uma estrutura dotada de um núcleo irredutível de hipóteses básicas das teorias levantadas, protegido por um cinturão protetor de hipóteses auxiliares e por heurísticas positivas e negativas que guiam o processo de investigação científica, é detalhadamente explicada. Além disso, os critérios demarcacionistas para o autor, relacionados com o progresso da ciência, são explicados com rigor.

Na terceira parte do trabalho, são apontadas as principais tentativas de aplicação da obra de Lakatos na economia neoclássica. De acordo com Hands (1992), a literatura sobre as ideias de Imre Lakatos associadas à ciência econômica pode

1 De acordo com Chalmers (1981, p. 113), "Lakatos desenvolveu sua descrição da ciência como uma tentativa de melhorar o falsificacionismo popperiano e superar as objeções a ele". 
ser dividida em duas categorias: a primeira traz as tentativas de aplicação dos conceitos lakatosianos para a interpretação de determinados episódios dentro da história do pensamento econômico; a segunda aborda as tentativas de aceitação da ideia dos programas de pesquisa como metodologia da ciência econômica, especialmente de maneira comparativa com as ideias de outros pensadores, como Popper e Kuhn. No presente trabalho, sobretudo são investigados os trabalhos de Weintraub (1985, 1979), que aplicou os conceitos lakatosianos à economia neowalrasiana teórica, os estudos de De Marchi (1976) e de Hendry (1993), aplicados à economia empírica, e o artigo de Blaug (1976), voltado à aplicação dos conceitos lakatosianos para a apreciação e diferenciação de programas de pesquisa na história do pensamento econômico.

Por fim, na quarta parte do trabalho são levantadas as principais críticas realizadas ao trabalho de Lakatos e suas aplicações à Economia. Segundo se relata, essas críticas podem ser divididas entre discordâncias voltadas ao próprio pensamento de Lakatos, discordâncias ao falsificacionismo que o inspirou e discordâncias aos próprios objetivos da metodologia da ciência que orientou a obra do autor.

\section{Filosofia e Metodologia da Ciência em Imre Lakatos}

Para Lakatos (1978), a unidade primordial de estudo e análise na filosofia da ciência não são teorias isoladas, mas sim os programas de pesquisa. O autor define como um programa de pesquisa uma série, isto é, um conjunto de teorias científicas afins por sua heurística e por um núcleo de hipóteses básicas que orienta a pesquisa científica, positivamente e negativamente, por um conjunto de regras aceitas por todos os cientistas individuais que aderem ao programa. Chalmers (1981) caracteriza a definição lakatosiana de programa de pesquisa como uma estrutura, na qual as teorias científicas são vistas como partes de um todo estruturado.

Lakatos afirma que cada programa de pesquisa apresenta um núcleo irredutível, ${ }^{2}$ composto por uma série de suposições básicas que são aceitas como irrefutáveis pelos cientistas integrantes do programa. Em outras palavras, o núcleo irredutível contém os pressupostos metafísicos fundamentais ao programa, ou seja, que definem o programa. Desse modo, participar de um determinado programa de pesquisa significa, para os cientistas, aceitar e ter seu trabalho guiado pelo núcleo irredutível. Segundo Chalmers (1981, p. 113), “[...] o núcleo irredutível de um programa é, mais do que qualquer outra coisa, a característica que o define. Ele assume a forma de uma hipótese teórica muito geral que constitui a base a partir

2 O termo original no inglês é hard core, e foi traduzido na literatura em língua portuguesa como "centro firme", "núcleo duro", "núcleo firme" ou "núcleo irredutível". No presente trabalho, optou-se pela última tradução, proposta em Chalmers (1981). 
da qual o programa deve se desenvolver". Ou seja, o núcleo de um programa de pesquisa é um conjunto de teorias ou hipóteses infalsificáveis no sentido popperiano. Para defender seu núcleo irredutível de possíveis anomalias observadas, cada programa de pesquisa conta com um cinturão protetor, uma heurística positiva e uma heurística negativa que orientam os processos de pesquisa.

O cinturão protetor consiste em um conjunto de suposições adicionais ao núcleo irredutível que compõem a estrutura do programa de pesquisa, protegendo-o de qualquer anomalia ou problema encontrado ao longo do trabalho científico. Chalmers (1981) aponta que o cinturão protetor não consiste apenas em um conjunto de hipóteses adicionais ao núcleo, mas também em proposições de testes de significância e de observações e em descrições das condições iniciais assumidas pelas hipóteses levantadas. Ao contrário do núcleo irredutível, as afirmações incluídas no cinturão protetor podem ser falsificadas e substituídas por outras mais capazes de defender o núcleo. Nas palavras do próprio Lakatos (1978, p. 91):

Chamo a essa cintura uma cintura protetora porque ela protege o centro firme das refutações: as anomalias não são encaradas como refutações do centro firme, mas de uma hipótese da cintura protetora. Em parte, por influência da pressão empírica (mas, em parte, respeitando um plano definido pela sua heurística), a cintura protetora é constantemente modificada, melhorada, complicada, enquanto o núcleo firme permanece intacto.

Cada programa de pesquisa apresenta uma heurística negativa que orienta o procedimento de um trabalho científico, de modo que o núcleo irredutível do programa seja mantido, isto é, não refutado, não modificado e não afetado por qualquer anomalia observada. A heurística positiva, por sua vez, orienta como pode ser desenvolvido um programa de pesquisa pela inclusão de suposições adicionais ao seu núcleo, assim como de técnicas de mensuração matemática, de observação empírica e de testes, de modo a explicar fenômenos passados e prever fenômenos futuros. Em outras palavras, a heurística positiva assume a proposição de que, para que um programa de pesquisa seja capaz de explicar e de prever fatos, é preciso que o seu núcleo irredutível seja complementado, e que o seu cinturão protetor seja constantemente alterado de modo a superar qualquer anomalia encontrada ao longo do trabalho científico. ${ }^{3}$

Para Lakatos, o mérito de um programa de pesquisa está relacionado com o seu caráter dinâmico, isto é, com o comportamento do programa frente a novas descobertas e novos problemas observados pelo processo de investigação cientí-

3 Nas palavras do próprio Lakatos (1978, p. 31), a heurística positiva de um programa de pesquisa "[...] define os problemas, esboça a construção de uma cintura de hipóteses auxiliares, prevê anomalias e transforma-as vitoriosamente em exemplos, tudo de acordo com um plano pré-concebido. O cientista registra as anomalias, mas desde que o seu programa de investigação agüente o seu ímpeto, ele pode simplesmente pô-las de parte". 
fica. Isso inclui, para o programa, dois fatores básicos. Em primeiro lugar, um programa de pesquisa deve manter um grau de coerência de suas heurísticas capaz de orientar harmonicamente a investigação científica futura por parte dos seus integrantes. Em segundo lugar, o programa de pesquisa não pode se limitar a explicar fatos passados, mas deve ser capaz de prever fatos novos. Logo, o programa deve ser progressivo.

O autor estabelece que um programa é teoricamente progressivo se as modificações realizadas no seu cinturão protetor, ao longo do processo de investigação científica e observação de anomalias, permitem previsões de fatos novos. Se, além disso, essas novas previsões forem confirmadas pelas evidências empíricas (observações), o autor define o programa como empiricamente progressivo. Por outro lado, um programa de pesquisa é visto como estagnado, ou degenerativo, se as modificações no cinturão protetor adotadas servem apenas para explicar as anomalias encontradas, sendo o programa incapaz de prever novos fatos. Lakatos (1978, p. 33) apresenta a seguinte diferenciação entre programas de pesquisa progressivos e estagnados:

Diz-se que um programa de pesquisa está a progredir enquanto o seu desenvolvimento teórico antecipar o seu desenvolvimento empírico, ou seja, enquanto ele continuar a predizer fatos novos com algum sucesso (alteração de problemas progressiva); ele estagna se o seu desenvolvimento teórico ficar para trás do seu desenvolvimento empírico, ou seja, enquanto fornecer somente explicações post hoc tanto de descobertas ocasionais como de fatos antecipados e descobertos no seu seio por um programa rival (alteração de problemas degenerativa). Se um programa de investigação explicar progressivamente mais do que um seu rival, suplantá-lo-á, e o rival pode ser eliminado (ou, se preferirem, arquivado).

Ou seja, Lakatos coloca que os programas de pesquisa progressivos acabam superando os seus rivais estagnados, em um sistema de competição entre os mesmos. É possível estabelecer um paralelo entre as ideias do autor e a noção das revoluções científicas e dos paradigmas de Thomas Kuhn. Contudo, é importante ter em mente que, para Lakatos, a revolução científica, entendida como um processo de substituição de programas de pesquisa estagnados por programas progressivos, não é um fenômeno de psicologia social, como para Kuhn (1982), mas sim um fenômeno histórico e racional. Isto é, o autor aponta que os cientistas racionalmente preferem trabalhar em um programa de pesquisa com a possibilidade de realizar novas descobertas do que naqueles programas estagnados. De acordo com Backhouse (1993, p. 175): 
The Lakatosian account of how one programme supersedes another places greater emphasis on rationality than does Kuhn's account of paradigm-shifts, and the irrational, "gestalt-shift" aspect of the process is completely absent. In both the Kuhnian and Lakatosian frameworks, however, the main force for change is the need to modify theoretical frameworks to take account of anomalies and deal with new problems.

O caráter histórico das revoluções científicas para Lakatos está representado pela inexistência de experimentos cruciais que possam refutar um programa de pesquisa inteiro de uma única vez. Segundo o autor, a maleabilidade do cinturão protetor ajuda a absorção das anomalias observadas pela investigação e isso torna os cientistas mais rígidos em sua cooperação com um determinado programa de pesquisa. Nesse ponto, a visão de Lakatos aproxima-se da de Kuhn. Contudo, Lakatos faz uma especial ênfase em um retorno ao demarcacionismo popperiano. Isto é, ao contrário de Kuhn, Lakatos procura meios de comparação entre "boa ciência", "pseudociência" e "não ciência" na dinâmica das revoluções científicas.

Tal ponto é explorado em sua visão sobre a metodologia do trabalho científico. Segundo Lakatos, o trabalho no interior de um determinado programa de pesquisa consiste em explicar e modificar a estrutura de seu cinturão protetor, de modo a alterar as hipóteses levantadas para resolver os problemas encontrados ao longo da investigação científica. Preocupado com o procedimento de uma "boa ciência", Lakatos definiu que os movimentos realizados no cinturão protetor não deveriam ser ad hoc, isto é, as novas hipóteses constantemente levantadas pelos cientistas devem ser passíveis de serem testadas individualmente. Ou seja, agindo dessa forma, os cientistas permitem que seus trabalhos sejam testados, e possivelmente falsificados e refutados, o que permite o progresso da ciência como um todo. Hands (1993) aponta essa ideia levantada por Lakatos como o progresso heurístico de um programa de pesquisa.

Comparando-se o pensamento de Lakatos com o de Popper (1996), que assumidamente foi o seu inspirador, observa-se que ambos concordam na importância do conteúdo empírico para as teorias científicas. Isto é, para ambos, a relação entre fatos observados e teorias levantadas tem um caráter falsificacionista. O conteúdo empírico de uma teoria científica é um conjunto de observações que podem detectar anomalias, as quais promovem o progresso científico. Contudo, Lakatos discorda de Popper ao defender que o núcleo irredutível de um programa de pesquisa é imune a essas anomalias e ao próprio falsificacionismo. 
Além disso, Lakatos faz uma maior ênfase nos fatos novos para a demarcação de uma "boa ciência", isto é, a capacidade de previsão é uma melhor demarcação de ciência progressiva do que a possibilidade de falsificação de suas hipóteses, como para Popper. ${ }^{4}$

\section{Tentativas de Aplicação dos Programas de Pesquisa Lakatosianos na Eco- nomia Neoclássica}

O trabalho de Imre Lakatos sobre a metodologia da ciência foi publicado a partir do início dos anos 1960 e foi rapidamente apreciado pelos metodólogos da ciência econômica, como Latsis (1976). Hands (1993, 1992) cita três fatores principais pelos quais a metodologia dos programas de pesquisa, proposta por Imre Lakatos, parece agradar aos economistas. Em primeiro lugar, pela observação de que a teoria econômica, em todos os seus paradigmas alternativos, apresenta núcleos irredutíveis de hipóteses metafísicas, sendo que as diferenças dessas hipóteses demarcam esses próprios paradigmas. ${ }^{5}$ Em segundo lugar, pelo fato de que a metodologia lakatosiana é mais próxima do trabalho de investigação científica na economia do que o falsificacionismo popperiano. Isto é, muitos economistas concordam que o falsificacionismo não é compatível com a ciência econômica contemporânea, já que nem todas as hipóteses iniciais assumidas pelas teorias têm condições de serem testadas empiricamente de modo isolado. Inclusive, como já mencionado, muitas delas têm caráter metafísico. Contudo, nem por isso a economia deixa de ser uma ciência empírica. Os fatos observados e a análise de dados são fundamentais para a análise econômica. O lado empírico da ciência econômica é mais complexo do que o proposto pelo falsificacionismo popperiano e se aproxima mais da visão lakatosiana de que as hipóteses centrais de um programa de pesquisa estão protegidas de testes empíricos pelas heurísticas do mesmo. Por fim, o autor aponta a importância da metodologia lakatosiana para a construção de estudos na história do pensamento econômico. Isto é, para o autor, o desenvolvimento histórico da economia pode ser estudado mediante a identificação e

$4 \quad$ Outras diferenças entre as teorias dos dois autores são descritas por Hands (1993, p. 67): "While Lakatos defines empirical content in a thoroughly Popperian way, he has no respect for the role os falsification in science. For Lakatos all theories are 'born refuted' [...] and the task of philosophy of science should be to develop a methodology which starts from this fact. For Lakatos progress comes from the corroboration not falsification of novel facts. Finally, Lakatos clearly embraces a historical meta-methodology whereby the actual history of science is used to appraise various methodological proposals".

5 Hands $(2001,1992)$ cita artigos nos quais a metodologia lakatosiana de programas de pesquisa é aplicada na economia clássica (PETRELLA, 1988), neoclássica (FISCHER, 1986), keynesiana (BLAUG, 1991, 1976; LEIJONHUFVUD, 1976), pós-keynesiana (BROWN, 1981), novo clássica (MADDOCK, 1974), austríaca (RIZZO, 1982) e marxista (BLAUG, 1980). 
a diferenciação de programas de pesquisa e o uso dos conceitos lakatosianos de núcleo irredutível, heurísticas e progresso/estagnação para diferenciá-los. ${ }^{6}$

Dentre as tentativas de aplicar diretamente a metodologia lakatosiana para a teoria econômica neoclássica, destacam-se os trabalhos de Weintraub (1988, 1985, 1979). ${ }^{7}$ Esse autor procurou utilizar os conceitos da metodologia dos programas de pesquisa para identificar a estrutura da teoria do equilíbrio geral walrasiano. $\mathrm{O}$ autor denominou o programa de pesquisa abordado como economia neowalrasiana e identificou o seu núcleo irredutível e o seu cinturão protetor. As evidências empíricas testariam as possibilidades de aplicação do equilíbrio geral, ${ }^{8}$ mas não as suas hipóteses básicas. Segundo Weintraub (1988, p. 166-167):

The hard core suppositions are: HC1. There exist economic agents; HC2. Agents have preferences over outcomes; HC3. Agents independently optimize subject to constraints; $\mathrm{HC} 4$. Choices are made in interrelated markets; HC5. Agents have full relevant knowledge; HC6. Observable economic outcomes are coordinated, so they must be discussed with reference to equilibrium theories. And the positive and negative heuristics are: $\mathrm{PH} 1$. Go forth and construct theories in which economic agents optimize; PH2. Construct theories that make predictions about changes in equilibrium states; $\mathrm{NH} 1$. Do not construct theories in which irrational behavior plays any role; NH2. Do not construct theories in which equilibrium has no meaning; NH3. Do not test the hard core propositions.

Ou seja, segundo esse estudo, a heurística positiva do programa de pesquisa neowalrasiano consiste na definição de técnicas para a construção de teorias e modelos que assumem a presença de agentes otimizadores e que são capazes de prever o ponto de equilíbrio de mercado. Isto é, induz o pesquisador a construir teorias baseadas no comportamento otimizador de agentes econômicos e sua implicação em termos de resultados em posição de equilíbrio, permitindo a exploração de diferentes interpretações para termos como "agente", "produto", "informação" e "mercados" (WEINTRAUB, 1988). Já a heurística negativa orienta a não teorização de modelos com irracionalidade, ou nos quais o equilíbrio de

6 Nas palavras do autor (HANDS, 1993, p. 68), “[...] economists have recently been very sympathetic to methodological proposals that are sensitive to the actual history of their discipline. Economists have produced an extensive literature using the Lakatosian categories to reconstruct various parts of the history of economic thought. Most of this literature focuses on a particular research programme in economic theory (past or present) and tries to isolate the hard core, the positive and negative heuristics, and the type of theoretical activity ocurring in the protective belt. Such work usually results in a positive or negative Lakatosian appraisal of the 'progessivity' of the particular economic research programme. Examples of these reconstructions range widely over various topics in the history of economic thought".

$7 \quad$ Esse trabalho é muito bem comentado por Hands (2001) e por Weiss (2002).

8 O autor cita como exemplos a teoria do capital humano, a economia da família e as teorias do comércio internacional. 
mercado é irrelevante, ou, ainda, tentativas de testar empiricamente as hipóteses básicas do núcleo irredutível.

Outro ponto destacado pelo autor é a importância da linguagem matemática nessa teoria. Segundo ele, a matematização da teoria do equilíbrio geral walrasiano se justifica por dois pontos básicos: o primeiro pelo processo de crescente refinamento sistemático da interpretação das hipóteses que integram o seu núcleo irredutível e que se baseia em uma procura por cada vez maior consistência lógica; e o segundo pelo fato de que o próprio equilíbrio geral é um fenômeno matemático e deve ser representado como tal. Weintraub (1985) observa que, pelo crescente refinamento da interpretação das hipóteses do núcleo por técnicas matemáticas, e pela expansão de seu cinturão protetor mediante o desenvolvimento de teorias que aplicam a noção de equilíbrio geral e que são passíveis de teste empírico, pode-se concluir que a teoria do equilíbrio geral apresenta progresso teórico. Se as teorias que compõem o cinturão protetor não forem empiricamente descartadas, pode-se ainda concluir que a economia neowalrasiana como um todo é progressiva no sentido lakatosiano do termo.

Outro autor que procurou aplicar a metodologia dos programas de pesquisa na ciência econômica foi De Marchi (1991). Segundo esse autor, os programas de pesquisa lakatosianos atraem a simpatia de muitos economistas neoclássicos por dois motivos: primeiro, porque a ciência econômica é considerada racional, então isso pode identificá-la como progressiva lakatosianamente. Segundo, porque as teorias que compõem a economia neoclássica são estruturadas, de modo que não são passíveis de serem analisadas individualmente, tal como Lakatos aponta em seus programas de pesquisa.

Ou seja, a metodologia lakatosiana parece defender o trabalho dos economistas contemporâneos (DE MARCHI, 1991), que é procurar explicar e prever uma grande série de problemas econômicos utilizando poucos pressupostos comportamentais para a ação dos agentes, definidos como a sua racionalidade. ${ }^{9}$ Além disso, esses pressupostos comportamentais são assumidos, não sendo passíveis de testes empíricos.

Latsis (1972), também procurando relacionar o método da economia neoclássica com a metodologia científica de Imre Lakatos, sugeriu que o núcleo do

$9 \quad$ Nas palavras de Backhouse (1993, p. 180), “[...] economists find Lakatosian methodology attractive because it provides a way of defending wat they do, This arises from the nature os economic theorizing as it exists in the mainstream of economics today. Economic theory is dominated by the attempt to explain a variety of economic phenomena on the basis of a very limited range of behavoral assumptions. Explaining a phenomenon involves demonstrating how it follows from the assumption of rational behaviour, any other assumption being viewed as ad hoc, for agents ought to behave rationally. Furthermore, given that assumptions cas rarely be tested directly (experimental work being both problematic and in its infancy, and econometric testing frequently being inconclusive) the only option open to economists wishing to test theories is to derive further predictions which can be compared with other evidence". 
programa de pesquisa da economia mainstream é o que o autor chamou de determinismo situacional. Segundo essa hipótese, os indivíduos presentes na teoria econômica apresentam um único comportamento, que é o de reagir aos incentivos presentes em cada situação. ${ }^{10}$ Explicando melhor, de acordo com Mäki (2006, p. 3),

According to situational determinism, the situation in which an agent finds itself determines its behaviour. The inner workings of the agent do not matter, as the external situation completely constrains its behaviour. The agent's situation is construed so as to leave it with no choice: in a single-exit situation, there is just one way to go, so there is no room for genuine choice, decision-making, and entrepreneurship. Behaviour becomes a matter of reaction instead of action.

Uma tentativa de aplicação da metodologia lakatosiana à economia empírica é realizada por Hendry (1993). Segundo o autor, a metodologia lakatosiana fornece uma estratégia de investigação científica, pois enfatiza a previsão de fatos novos. ${ }^{11} \mathrm{Ou}$ seja, o que realmente interessa é que as teorias formuladas e assumidas funcionem, e não que sejam realistas e falsificáveis. Assim, um programa de pesquisa na economia deve ter três objetivos: primeiro, realizar previsões de fatos novos; segundo, explicar mais fenômenos do que os programas rivais, em um ambiente científico competitivo; terceiro, demonstrar novas conexões entre fenômenos econômicos, seguindo o que Lakatos denominou como progresso teórico do programa.

A proximidade entre a metodologia lakatosiana e a economia empírica também é destacada por Backhouse (1993, p. 181):

[...] Lakatosian ideas are expressed by economists deeply committed to economics being an empirical science, driven by data. Prediction of novel facts is used, especially by Hendry, not because it is a soft option, but because it is both feasible (in a way that naïve falsificationism is not) and demanding. [...] prediction of novel facts has a history in economics that goes back well before Lakatos. It is plausible to conjuncture that economists found Lakatos attractive because the appraisal criterion he used was already, perhaps for good reasons, well established.

10 Um ponto polêmico no trabalho de Latsis (1972) é que o autor considera que a economia neoclássica, definida pela hipótese do determinismo situacional, consiste em um programa de pesquisa degenerativo, cujo progresso teórico é incapaz de gerar novas previsões empíricas, e serve apenas para corrigir suas próprias limitações. Assim, para o autor, esperaria-se que os pesquisadores na economia migrassem para campos de pesquisa novos, de futuro mais promissor.

11 Nesse ponto, a visão de Hendry aproxima-se bastante do trabalho clássico de Friedman (1953). Por outro lado, distancia-se de algumas das ideias do próprio Lakatos, para quem o realismo das proposições científicas é considerado importante. 
Segundo De Marchi (1991) e Hands (1993), a ênfase na previsão de fatos novos para a definição do progresso científico é o maior legado de Lakatos para a economia empírica por três motivos. Em primeiro lugar, essa visão ajusta-se ao próprio trabalho dos economistas, já que, pelo menos na economia neoclássica, na ausência de dados para testar todas as hipóteses assumidas, procura-se a consistência dessas hipóteses com o critério da racionalidade dos agentes, o que decide se essas teorias são ad hoc ou não. Em segundo lugar, a procura por previsões confiáveis é o principal trabalho de economistas profissionais, já que, para a tomada de decisões econômicas (como de política econômica), os resultados devem ser conhecidos e esperados. Nesse sentido, o autor aponta que Lakatos diferencia os tipos de previsões que um programa de pesquisa pode realizar no que diz respeito a fatos novos, que podem ser tanto fatos desconhecidos para o pesquisador, quanto também fatos desconhecidos para a própria teoria à luz da qual a previsão é realizada. Por fim, como na economia não há a possibilidade de se realizar experimentos controlados para isolar fenômenos e associar teorias com os fatos observados, a capacidade de previsão, conforme proposto por Lakatos, torna-se um importante método de se testar a qualidade das teorias, sejam novas, sejam as mais aceitas no presente. Ou seja, segundo o autor, uma teoria mostra sua qualidade quando é capaz de realizar previsões, mesmo quando é aplicada a novas situações.

Backhouse (1993) ainda destaca a importância que a metodologia desenvolvida por Lakatos dá à relação entre a história e a filosofia da ciência. Tal visão aponta uma nova função para a metodologia da ciência, isto é, a procura de explicações para o desenvolvimento histórico de teorias científicas. Segundo o autor, essa função envolve as seguintes tarefas:

Lakatos's MHRP involves the following four stages: (1) Obtain agreement on a list os successful scientific achievements. (2) Provide a history of these scientific achievements as though they had developed in accordance with the methodology one is trying to appraise - what Lakatos calls a 'rational reconstruction' of history. (3) Compare this rational reconstruction with the actual history. (4) If the two histories are very different, conclude that the methodology is inappropriate: that it is incompatible with the decisions made by practicing scientists (BACKHOUSE, 1993, p. 184).

Nesse mesmo sentido, Hausman (2004) aponta uma contribuição de Lakatos para solucionar o que esse autor chama de "Problema de Mill", permanentemente em discussão na economia. Segundo Hausman, esse problema consiste na possibilidade de se poder tomar deduções corretas a partir de premissas não observáveis, não testáveis, ou mesmo falsas. Nesse sentido, "Lakatos appears to solve Mill's problem by arguing that what matters is empirical progress or retrogression rather than empirical success or failure" (HAUSMAN, 2004, p. 9). Assim, as hipóteses bá- 
sicas da economia neoclássica, que não são testáveis empiricamente, mas sempre são tidas como aceitas nos trabalhos científicos dessa linha de pesquisa, acabam sendo associadas ao núcleo irredutível de um programa de pesquisa. ${ }^{12}$

Uma das mais conhecidas tentativas de aplicação da metodologia dos programas de pesquisa, proposta por Lakatos, para a descrição da história do pensamento econômico é o trabalho realizado por Blaug (1976). O autor, em resumo, procurou comparar os paradigmas dentro da ciência econômica ao longo de seu desenvolvimento histórico demarcando-os como programas de pesquisa e caracterizando-os utilizando os conceitos desenvolvidos por Lakatos. Por exemplo, ao comparar a economia política clássica e a economia positiva neoclássica como programas de pesquisa, o autor observou que o núcleo irredutível de ambos (presença de noção de equilíbrio, comportamento otimizador por parte dos agentes) é muito semelhante. O que diferencia os dois programas seria a heurística positiva, a ênfase da investigação científica: enquanto que na economia clássica os economistas voltam sua atenção à produção e distribuição de riqueza em uma sociedade, na economia neoclássica o foco de interesse é o funcionamento dos mercados, isto é, a alocação de recursos escassos entre fins alternativos.

Por outro lado, a revolução keynesiana, na década de 1930, afetou o núcleo irredutível da ciência econômica e definiu uma nova heurística positiva e um novo cinturão protetor. Por isso, o autor identificou a revolução keynesiana como uma revolução científica lakatosiana, pela superação de programas de pesquisa estagnados por programas progressivos. ${ }^{13}$

Um outro estudo aplicado relacionando os programas de pesquisa de Lakatos e a ciência econômica foi realizado por De Marchi (1976) e denominado "O Paradoxo de Leontief”. Esse estudo proveio de uma anterior investigação empírica realizada por Leontief, que pareceu falsificar a teoria do comércio internacional de Hecker-Ohlin, ${ }^{14}$ bastante aceita pelos economistas neoclássicos. De Marchi procurou observar como a comunidade internacional de economistas reagiu a essa

12 "The fact that economists do not give up basic theoretical postulates that appear to be false might be explained and justified by regarding them as part of the 'hard core' of the neoclassical research programme" (HAUSMAN, 2004, p. 9).

13 Na verdade, nesse ponto de seu trabalho, Blaug (1976) procurou comparar os programas de pesquisa lakatosianos com os paradigmas kuhnianos para verificar qual seria a melhor metodologia para descrever o desenvolvimento histórico do pensamento econômico. Como o autor observou que a revolução keynesiana não suplantou totalmente o paradigma da economia neoclássica, sobretudo porque essa teoria não consiste em um paradigma unificado, mas sim em um conjunto de teorias interdependentes configuradas em um programa de pesquisa, concluiu que a visão lakatosiana seria mais apropriada para esse fim.

14 Segundo essa teoria, os países, operando em livre-comércio internacional, tendem a se especializar na produção e exportação de bens compatíveis com a abundância interna relativa de fatores de produção. Isto é, países intensivos em capital tendem a se especializar em produtos cuja produção é intensiva em capital, e o mesmo vale para outros bens. O estudo de Leontief, que contrastou essa teoria, obsevou que, apesar da economia norte-americana ser intensiva em capital, a pauta de exportações do país é baseada em bens cuja produção é intensiva em trabalho. 
conclusão. Segundo o autor, a maioria dos estudiosos procurou defender a teoria e atacar o estudo de Leontief, ou seja, a teoria não foi descartada mesmo com uma evidência empírica contrária a ela. De Marchi chegou à conclusão de que os economistas tiveram um comportamento tal como esperado para integrantes de um programa de pesquisa lakatosiano, ao qual essa teoria do comércio internacional é parte de seu núcleo irredutível, tornando-se relutantes em abandoná-lo ou modificá-lo frente a uma anomalia detectada.

\section{Críticas à Metodologia Lakatosiana na Ciência Econômica}

Conforme destacado por Backhouse (1993), e confirmado por toda a bibliografia, após um surto inicial de otimismo durante a segunda metade da década de $1970,{ }^{15}$ a partir dos anos 90 o interesse geral dos economistas pela metodologia dos programas de pesquisa lakatosianos caiu bruscamente. Os trabalhos dirigidos a investigar correlações entre a metodologia lakatosiana e a metodologia da economia neoclássica como é feita realmente deixaram de ter um tom tão entusiasta, e passaram a ser mais críticos. De acordo com Backhouse (1993), todas as críticas realizadas aos programas de pesquisa de Lakatos podem ser reunidas em três grupos: no primeiro, as críticas realizadas diretamente aos conceitos e definições levantados pelo autor; no segundo, as críticas realizadas ao método falsificacionista como um todo, o qual influenciou e inspirou Lakatos; e, no terceiro, as críticas realizadas à própria metodologia da ciência e à tentativa de generalizar teorias que descrevem o processo de geração de conhecimento e de procurar demarcar aquilo que pode ser considerado "boa ciência" das chamadas "pseudociências" e "não ciências".

\subsection{Críticas a Lakatos}

Uma primeira crítica que pode ser realizada aos conceitos levantados por Imre Lakatos refere-se à visão do autor sobre a presença, em cada programa de pesquisa, de um núcleo irredutível de hipóteses básicas fixas e não alteráveis entre os cientistas. De acordo com Hoover (1991) e Backhouse (1993), tal argumento não procede na ciência econômica, já que é observável que os chamados núcleos das principais correntes de pensamento econômico são dinâmicos, variando ao longo do tempo. Por isso, a relação entre o programa de pesquisa e o seu núcleo irredutível, que o caracterizaria na visão de Lakatos, é bem mais complexa na economia do que o autor originalmente supunha.

15 Principalmente após a publicação da coletânea de artigos sobre a metodologia da ciência econômica por Spiro Latsis, em 1976, que explicitamente procurou paralelos entre esse tema e as ideias de Lakatos. 
Outra fonte de críticas, por diversos autores, são os trabalhos de Weintraub (1985, 1979). Segundo Backhouse (1993), o que o autor originalmente definiu como o núcleo irredutível da economia neowalrasiana - a hipótese do comportamento otimizador dos agentes econômicos guiado pela sua racionalidade - consiste, na verdade, mais em uma questão metodológica para a modelagem de teorizações do que em hipóteses metafísicas com conteúdo econômico significante. Hands (2001) faz uma crítica ainda mais aguda a esse trabalho. Segundo esse autor, não fica esclarecido no estudo original de Weintraub se o equilíbrio geral walrasiano é o núcleo de um programa de pesquisa próprio, ou se consiste em um cinturão protetor de um programa de pesquisa neoclássico mais genérico. Além disso, apontar como observação de progresso teórico no programa de pesquisa neowalrasiano o simples progresso da formalização matemática de suas hipóteses não é uma concordância unânime entre os estudiosos do pensamento de Lakatos. ${ }^{16}$ Além disso, a noção de que o progresso empírico do mesmo programa de pesquisa pode ser comprovado pela observação do desenvolvimento de outras teorias econômicas que aplicam a noção de equilíbrio geral parece seguir uma visão mais positivista e popperiana do que propriamente lakatosiana, pois, para Lakatos, o progresso empírico de um programa de pesquisa está relacionado com a sua capacidade de realizar previsões empiricamente significantes de fatos novos, isto é, fatos descobertos, e não apenas refinamentos da própria teoria. Mais ainda, o trabalho de Weintraub não aponta explicitamente qual seria o cinturão protetor do programa neowalrasiano e tampouco como esse cinturão se relaciona com o núcleo irredutível, protegendo-o de refutações empíricas.

No entanto, a principal crítica que pode ser realizada ao estudo, na visão de Hands (2001), é o fato de que Weintraub utiliza a metodologia lakatosiana para justificar a teoria do equilíbrio geral walrasiano como um programa de pesquisa progressivo. Todavia, a grande maioria dos metodólogos de visão lakatosiana presentes na ciência econômica são exatamente contra essa noção. Segundo eles, a economia é uma ciência empírica, e o equilíbrio geral, por ser um instrumental matemático abstrato, é muito pouco empiricamente testável, o que não o pode tornar um programa de pesquisa empiricamente progressivo no sentido lakatosiano do termo. ${ }^{17} \mathrm{Tal}$ conclusão levou muitos dos economistas simpatizantes da metodologia lakatosiana a rever os seus conceitos e a tomar uma atitude mais crítica e realista em relação a esse autor. De acordo com Blaug (1992, p. 169),

16 Mäki (2006) sugere que o avanço da formalização matemática na economia seja entendido como um "progresso dedutivo", e não um progresso teórico ou empírico.

17 "For these authors, the problem with economics lies in its lack of empirical discipline - falsificationism is a little too strict in practice, but its empirical spirit is right - Lakatos is desirable because his approach allows us to keep much of modern economics (which a strict application of falsificationism would force us to condemn as uncientific), while still providing tough empirical rules for scientific progress in economics" (HANDS, 2001, p. 294). 
Enormous intellectual resources have been invested in its endless refinements, none of which has even provided a fruitful starting point from which to approach a substantive explanation of the workings of economic system. Its leading characteristic has been the endless formalization of purely logical problems without the slightest regard for the production of falsiable theorems about actual economic behavior, which, we insist, remains the fundamental task of economics. The widespread belief that every economic theory must be fitted into the GE mold if it is to qualify as rigorous science has perhaps been more responsible than any other intellectual force for the purely abstract and nonempirical character of so much of modern economic reasoning.

Seguindo essa mesma linha, Hausman (1994) aponta que a definição da estrutura de um programa de pesquisa na economia é uma atividade complexa. Segundo o autor, para se encontrar o núcleo irredutível, o cinturão protetor e as heurísticas de qualquer programa de pesquisa que possa ser identificado dentro da ciência econômica não basta utilizar apenas a observação, mas também é preciso ajustar a própria teoria econômica aos conceitos lakatosianos, e essa tarefa nunca foi bem explicada por estudos. Por exemplo, o próprio trabalho de Weinstraub (1985) utiliza uma construção do núcleo irredutível neowalrasiano muito fraca, na visão de Hausman. Segundo esse autor, as hipóteses HC1, HC2 e HC4, apesar de verdadeiras para um núcleo de programa de pesquisa econômico, não diferenciam a economia neowlrasiana de outras correntes de pensamento. Por outro lado, de acordo com Backhouse (1991), as hipóteses HC5 e HC6 na verdade não consistem em um núcleo irredutível, já que muitos trabalhos de economia neoclássica utilizando o conceito de equilíbrio geral podem explorar teorias econômicas com a possibilidade de informação imperfeita entre agentes econômicos e desequilíbrios de mercado em curto prazo. Por isso, a única hipótese que inegavelmente consiste em um pressuposto inviolável da economia neowalrasiana é a HC3, relacionada ao comportamento dos agentes econômicos. Segundo essa hipótese, os agentes independentemente tomam decisões de modo a otimizar suas funções-objetivo, as quais estão sujeitas a quaisquer restrições impostas a eles. Entretanto, isso traz uma noção de que o núcleo irredutível desse programa de pesquisa é muito estreito. 
Segundo Hausman (1994, p. 204), os trabalhos da economia neoclássica podem variar muito em suas hipóteses assumidas, tornando muito complexa a identificação de um núcleo irredutível estável em seu programa de pesquisa: ${ }^{18}$

For there is very little that is shared by every neo-Walrasian theory or model. The hard core cannot include the claim that preferences are complete or transitive, for there are neo-Walrasian theoretical explorations which involve incomplete and intransitive preferences [...]. The hard core cannot include self-interest, for there are neo-Walrasian models with altruism. One cannot include diminishing returns, because there are neo-Walrasian models with fixed production coefficients. One cannot include profit maximization, for there are neo-Walrasian models [...] without profit maximization. If one insists on characterizing neo-Walrasian economics by those features shared by all neo-Walrasian theories and models, then one cannot do much better than Weintraub [...].

Complementando, Weiss (2002) aponta que definir um programa de pesquisa em qualquer paradigma dentro da economia é difícil, simplesmente porque Lakatos pensou sua teoria para a física e outras ciências exatas, e não para a economia. O autor reforça a ideia de que a definição de núcleos irredutíveis para a investigação econômica é problemática devido a dois fatos: em primeiro lugar, os programas de pesquisa, na economia, são interdependentes, e um pode estar contido no outro (como, por exemplo, a economia neoclássica e a economia novo-clássica); em segundo lugar, não se pode decidir com firmeza o tamanho exato de um programa de pesquisa na economia por causa da existência de sub-programas e pela dificuldade de se diferenciar núcleos e heurísticas de programas semelhantes. ${ }^{19}$

Outra crítica à aplicação da metodologia de programas de pesquisa na economia refere-se à possibilidade de sobreposição de programas de pesquisa nessa ciência. Por exemplo, Reder (1982) cita a comparação entre um programa de pes-

18 Hausman (1994, p. 205) procura identificar uma estrutura alternativa para a ciência econômica: " 1 Economics is defined in terms of the casual factors with which it is concerned, not in terms of a domain; 2 Economics has a distinct domain, in which its casual factors predominate; 3 The 'laws' of the predominating causal factors are already reasonably well known; 4 Economic theory, which employs these laws, provides a unified, complete, but inexact account of its domain". Segundo o autor, os fatores causais centrais na teoria econômica relacionam-se com a existência da escassez de recursos, e o seu domínio é o conjunto de escolhas racionais individuais sobre decisões de consumo e produção. O conceito de racionalidade não é fixamente definido na ciência econômica, significa apenas que há uma coerência entre as ações e as preferências individuais. $\mathrm{O}$ autor conclui que o único pressuposto que explicitamente governa a investigação econômica é o individualismo metodológico, isto é, a noção de que as decisões tomadas são provenientes de fenômenos individuais aos agentes, e não de grupos.

19 "Lakatos supplied a method of delineating programs, arguing that if a new theory did not include all of the unrefuted content of an earlier theory then it could be described as a separate research program. However, this distinction was inadequate to address a large literature in which one program's hard core contents may be the protective belt or positive heuristic of another." (WEISS, 2002, p. 15). 
quisa da economia neowalrasiana e um programa de pesquisa da economia da Escola de Chicago. ${ }^{20}$ Ou seja, programas distintos, ou mesmo rivais, podem ser indeterdependentes: o que é núcleo irredutível para um pode ser cinturão protetor ou heurística para o outro. As relações entre as diferentes correntes de pensamento econômico, fora a rivalidade explícita entre programas progressivos e estagnados, não é explicada apropriadamente pela metodologia de Lakatos.

No que diz respeito ao progresso empírico da ciência econômica, Hands (2001, 1991) e Hausman (2004) apontam que esse fator é muito duvidoso. Ou seja, a economia contemporânea é muito menos empírica do que aquilo que Lakatos idealizou que uma ciência deveria ser. Portanto, a ênfase do autor na descoberta e previsão de fatos novos para a apreciação de uma teoria científica não foi considerada por Hausman como uma solução satisfatória para o problema de Mill, referido no capítulo anterior do presente estudo.

Por outro lado, autores como Fawundu (1991) criticaram o uso da metodologia dos programas de pesquisa lakatosianos para a apreciação da evolução histórica de paradigmas de pensamento econômico, conforme realizado no artigo de Blaug (1976). Segundo Fawundu, Blaug acabou por distorcer os principais conceitos estabelecidos por Lakatos como forma de melhor ajustá-los ao objetivo de seu trabalho. Em primeiro lugar, Fawundu coloca que a heurística positiva, de acordo com o trabalho original de Lakatos, é sempre dinâmica, se ajustando conforme anomalias são descobertas no trabalho de investigação científica. Por isso, ao contrário do que Blaug apontou, a mudança da heurística positiva da economia política clássica para a economia positiva neoclássica não pode ser considerada um fator que indique uma revolução científica nessas condições. Em segundo lugar, a heurística positiva, de acordo com Lakatos, pode ser testada pelos fatos empíricos, o que pode levar inclusive a sua falsificação, refutação e substituição por novas hipóteses. Contudo, a história do pensamento econômico não sugere que houve uma refutação empírica da heurística clássica, ou mesmo que a heurística neoclássica, sobretudo o que diz respeito à teoria da utilidade marginal, possa ser testada e corrobada por fatos empíricos. Além disso, Fawundu aponta que, segundo Lakatos, a defesa apaixonada de um determinado programa de pesquisa frente aos seus rivais é um sinal evidente de sua estagnação.

Fawundu também aponta que, de acordo com o conceito lakatosiano de revolução científica, um programa de pesquisa progressivo acaba superando totalmente um rival estagnado. Porém, no caso entre o programa neoclássico e o

20 Segundo Backhouse (1993, p. 177), “[...] it cannot be argued that Chicago economics forms a sub-programme within neo-Walrasian economics: the commitment to formal modelling and mathematical rigour are missing. Friedman's 'Marshallian' methodology, stressing the importance of empirical evidence and simple models, is clearly not Walrasian. There is thus a strong case for speaking in terms of two overlapping programmes, with work conducted within one programme providing a crucial input into another programme". 
keynesiano, isso não aconteceu. Por um lado, o mainstream econômico logo após Keynes tornou-se uma síntese de seu pensamento com os antigos pressupostos neoclássicos. Por outro lado, os chamados economistas pós-keynesianos, considerados mais fiéis às teorias originais do autor, nunca chegaram a dominar debates na macroeconomia, tendo sempre sido considerados heterodoxos, isto é, contrários ao mainstream.

Outra crítica aos estudos que buscam descrever episódios da história do pensamento econômico utilizando as terminologias desenvolvidas por Imre Lakatos foi feita por Hands (1992). Segundo o autor, os pesquisadores que atuam nessa linha costumam tomar pouco cuidado com as terminologias lakatosianas. Isto é, há uma falta de fidelidade ao que Lakatos realmente queria dizer com seus conceitos de núcleo irredutível, heurísticas e, principalmente, previsão de fatos novos. Isso acontece, sobretudo, porque Lakatos desenvolveu esses conceitos pensando em programas de pesquisa aplicados nas ciências exatas.

A maior crítica que Hands (1993) faz a Lakatos deve-se a sua noção de progresso como forma de apreciação de teorias científicas. De acordo com o autor, esse progresso teórico e empírico, associado à capacidade de uma teoria científica ser capaz de prever fatos novos, de acordo com o sentido original do conceito levatado por Lakatos, é muito raro na economia. O progresso teórico na economia, conforme relatado por autores como Weinstraub (1985), se faz por maneiras distintas, como, por exemplo, pelo crescente refinamento da formaliação matemática das hipóteses levantadas pelas teorias. Essa visão é reforçada por Blaug (1991), segundo o qual a ênfase que Lakatos faz sobre a capacidade de prever fatos novos para considerar um dado programa de pesquisa progressivo acaba afastando-o da metodologia da economia, uma vez que pouco da teoria econômica contemporânea consegue de fato descobrir fatos novos.

Por outro lado, Hands (1993) chega a afirmar que a maior vantagem de se aplicar a metodologia lakatosiana dos programas de pesquisa na ciência econômica deve-se ao simples fato de que essa abordagem é mais verossímil do que a de Popper. Isto é, para o autor, Lakatos ajuda a aliviar a prática da investigação científica da economia aos critérios falsificacionistas, no sentido de que o aspecto empírico da ciência econômica, mesmo sendo de caráter fundamental, tem sua importância considerada de um modo mais complexo do que o tido pela visão popperiana. Assim, podem-se identificar núcleos de hipóteses não testáveis empiricamente nos paradigmas de pensamento econômico, assim como heurísticas e cinturões protetores de hipóteses testáveis, mesmo que sua identificação não seja simples e direta, conforme já relatado anteriormente.

Por fim, Gonzales (2001) critica o paralelo que não raramente se faz entre o instrumentalismo da teoria econômica, como o defendido por Friedman (1953), e a metodologia lakatosiana. De acordo com o autor, Lakatos não pode ser carac- 
terizado como um instrumentalista no que diz respeito a sua visão de filosofia da ciência, mas sim como um realista crítico. Lakatos defendeu que a capacidade de previsão de fatos novos era critério para o progresso de um programa de pesquisa, mas também considerou que uma teoria científica deveria ser capaz de explicar a realidade (ou os fatos passados) e as conexões entre diferentes fenômenos observados. Além disso, como um programa de pesquisa progride pelos seus avanços teóricos e empíricos, cada passo nesse progresso vai tornando as teorias científicas mais próximas da realidade, isto é, a heurística positiva do programa tende a simular a realidade com cada vez mais precisão. Por isso, Gonzales conclui que um programa de pesquisa, na visão de Lakatos, não tem a única utilidade de realizar previsões, mais também de, com seu progresso, aproximar o conhecimento humano da realidade. ${ }^{21}$

\subsection{Críticas ao Falsificacionismo e à Metodologia da Ciência}

Outra fonte de críticas à aplicação das ideias de Lakatos na ciência econômica tem foco na própria metodologia falsificacionista, que influenciou e baseou as teorias da metodologia dos programas de pesquisa.

Nessa linha, uma primeira crítica às limitações do falsificacionismo refere-se à ausência, nas discussões sobre a metodologia da ciência, de preocupação com o contexto em que as descobertas científicas são realizadas. De acordo com Backhouse (1993), Popper chegou até mesmo a considerar esse ponto irrelevante, afirmando que o contexto da descoberta é um fenômeno predominantemente psicológico, relacionado ao raciocínio e à criatividade do cientista, e sem relevantes dimensões de cunho filosófico.

Em outras palavras, a questão da sociologia da ciência, que envolve o estudo do contexto social nas quais as teorias científicas são criadas e apreciadas, foi subestimada e negligenciada pela metodologia falsificacionista. ${ }^{22}$ Contudo, Backhouse (1993) argumenta contra essa discriminação, por três motivos: o primeiro pelo fato de que o estudo dos métodos de se produzir teorias científicas é uma questão tão filosófica quanto a justificativa dessas mesmas teorias; o segundo porque o contexto social em que a criação de ideias se insere é fundamental para a sua divulgação e aceitação por parte da comunidade científica; e o terceiro, concordando com De Marchi (1992), mais particularmente no caso da ciência econômica, é porque o estudo do contexto social em que as teorias estão inseridas ajuda a se entender com mais profundidade as ideias que envolvem essas teorias:

$21 \quad$ Esse ponto é explorado por autores que criticam não apenas Lakatos, mas também o falsificacionismo metodológico como um todo.

22 Maiores detalhes sobre estudos que abordam o condicionamento social do comportamento dos cientistas podem ser encontrados em Mäki (1992). 
"For example, it is impossible to evaluate the results of econometric work without knowing the beliefs and convictions of the economists undertaking the work" (BACKHOUSE, 1993, p. 178).

Uma importante crítica sociológica à tentativa de aceitação dos programas de pesquisa lakatosianos à metodologia da economia neoclássica foi feita por Earl (1983). Segundo o autor, a noção de superação de programas de pesquisa tal como descrito originalmente por Lakatos é falha no que diz respeito à realidade da ciência econômica. Isto é, a "progressividade", isto é, a possibilidade de um programa de pesquisa prever fatos novos é insuficiente para que economistas migrem de uma corrente de pensamento para outra. Os economistas são, na visão do autor, muito mais conservadores e apegados a motivações individuais do que Lakatos originalmente supôs sobre o comportamento de cientistas racionais, uma vez que é observado que os economistas têm dificuldades em admitir que suas convicções estão erradas, mesmo diante de erros e de evidências contrários a elas. Para Earl, a escolha de se integrar a uma determinada corrente de pensamento econômico depende de três fatores: as predisposições individuais, que incluem as preferências e percepções pessoais, o contexto econômico social e acadêmico e a seletividade dos processos cognitivos.

Uma última fonte de críticas à visão de Imre Lakatos se dá a partir da possibilidade da filosofia da ciência poder levantar argumentos precisos para caracterizar e demarcar o que se pode considerar "boa ciência" e aquilo que seria "pseudociência" e "não ciência". Segundo autores como Backhouse (1993) e Hands (2001), não existem critérios filosóficos decisivos e infalíveis para qualificar teorias científicas, pois isso supõe que os filósofos já conhecem privilegiadamente uma noção precisa do que se pode considerar como "conhecimento verdadeiro". Além disso, autores como De Marchi (1992) e Mäki $(1992,2006)$ consideram que o conhecimento não é um conceito filosófico rígido, mas sim uma prática social, o que faz com que a investigação científica seja uma atividade socialmente construída.

Como alternativas para a metodologia demarcacionista, Backhouse (1993) cita correntes construtivistas e pós-modernistas referentes à filosofia da ciência exatamente por elas serem mais relativistas no que diz respeito à definição da qualidade de uma teoria científica.

Em síntese, pode-se concluir que a metodologia lakatosiana não é melhor do que a de Popper e nem do que a de nenhum outro demarcacionismo filosófico para poder decidir o que é "boa ciência" e o que não é. Como não existem critérios universalmente válidos para demarcar o que seria "boa ciência", isso faz com que cada tentativa sempre carregue uma alta dose de arbitrariedade. No caso lakatosiano, o "progresso" do programa de pesquisa só pode ser observado em uma perspectiva histórica quando os fatos novos que devem ser previstos já são conhecidos. No período presente, não se pode decidir quais programas de pesqui- 
sa vigentes são progressivos e quais estão estagnados simplesmente porque não se sabe quais os fatos novos que eles irão (ou mesmo se irão) descobrir.

Por outro lado, as premissas não estão satisfeitas na ciência econômica já que os economistas tendem a discordar entre si em questões metodológicas mesmo dentro de programas de pesquisa semelhantes. Além disso, a própria atividade profissional do economista, assim como os contextos individuais tende a distorcer as suas práticas de investigação científica pessoal, e não é possível descobrir qual economista utiliza a técnica que o permite se aproximar da realidade com maior precisão.

Por fim, Weiss (2002) destaca a importância dos estudos de filosofia e metodologia da ciência que dão importância para as convenções da linguagem e da retórica entre os cientistas. Segundo o autor, muitos dos problemas verificados no processo de investigação científica tendem a ser acomodados nos seus programas de pesquisa por meio do uso da retórica, isto é, por representações de ideias utilizando figuras de linguagem, e pelo uso de técnicas de cunho literário para defender e/ou criticar ideias. Tais práticas podem ser consideradas mais importantes do que discussões puramente metodológicas na filosofia da ciência.

\section{Considerações Finais}

O presente trabalho procurou fazer uma breve discussão das ideias de filosofia e metodologia da ciência em Imre Lakatos, assim como do seu contexto histórico dentro da filosofia da ciência, para então apresentar as propostas levantadas pela literatura de aplicação dessas ideias na metodologia da economia assim como suas críticas. Mesmo com a grande diversidade de opiniões e visões sobre as teorias do autor encontradas na bibliografia estudada, é possível tirar conclusões sólidas sobre as discussões abordadas.

Em primeiro lugar, observa-se o contexto filosófico em que o pensamento de Lakatos se insere. $\mathrm{O}$ autor procurou defender a metodologia científica falsificacionista popperiana, empírica e demarcacionista contra os ataques estruturalistas de Thomas Kuhn. De Kuhn, Lakatos incorporou a noção de que as teorias científicas se agrupam em estruturas (denominadas de paradigmas, para Kuhn, e programas de pesquisa, para Lakatos) e não são passíveis de apreciação individualmente. Além disso, Lakatos aceitou a rigidez da confiança e do comprometimento dos cientistas individuais com as teorias com as quais trabalham. Contudo, para o autor, ao contrário do que Kuhn apontava, essa rigidez demonstra um comportamento racional por parte dos indivíduos, os quais buscam se agregar àqueles programas de pesquisa que consideram progressivos. Contudo, como Backhouse (1993) muito bem observa, esses dois pontos não são os mais importantes da obra de Kuhn. A questão da sociologia da ciência, isto é, a noção de que o conhe- 
cimento científico é uma atividade socialmente construída e apreciada, é muito bem desenvolvida por Kuhn, mas é negligenciada por Lakatos. Isso, conforme a própria bibliografia explica, é uma grave limitação na obra desse autor, já que, principalmente na economia, as ideias presentes nas teorias levantadas tendem a estar relacionadas com o contexto histórico e social em que foram formuladas. Contudo, a metodologia de Lakatos pode ser considerada um avanço em relação ao falsificacionismo popperiano, predominante até então.

Em segundo lugar, o maior problema da metodologia de Lakatos, e que em grande parte explica o progressivo desinteresse da comunidade acadêmica pela obra do autor a partir dos anos 90, foi a sua insistência nos critérios demarcacionistas para a filosofia da ciência, presentes no falsificacionismo popperiano e no positivismo lógico. Atualmente, essa discussão já é tida como superada nos meios científicos, já que não há um critério universalmente válido que diferencie uma chamada "boa ciência" de uma "pseudociência" e que não esteja impregnada de arbitrariedade. O demarcacionismo acabou dando lugar a visões mais relativistas para a metodologia da ciência. Além disso, o próprio conceito de Lakatos para o mérito de uma ciência, relacionado ao seu progresso teórico e empírico, é problemático, sobretudo quando se tenta aplicá-lo na ciência econômica, a qual raramente apresenta novos fatos descobertos.

Por fim, apesar de suas falhas, a teoria de Lakatos não está descartada da ciência econômica na visão de muitos autores. Se, por um lado, o seu critério demarcacionista pode ser considerado abandonado, os demais conceitos lakatosianos, como o núcleo irredutível, as heurísticas, o cinturão protetor, ou, mesmo, o progresso científico, podem ter relevância para a apreciação de distintos paradigmas em estudos da história do pensamento econômico. Obviamente, deve-se controlar todos os problemas não previstos originalmente no pensamento do autor, como a sobreposição de programas de pesquisa e o aspecto dinâmico das hipóteses incluídas nos núcleos ditos irredutíveis. Contudo, mesmo sendo complexa, essa tarefa parece ser frutífera para trabalhos nessa área do conhecimento econômico.

\section{Referências}

BACKHOUSE, R. The Lakatosian legacy in economic methodology. In: BACKHOUSE, R. New directions in economic methodology. London: Routledge, 1993.

BACKHOUSE, R. The neo-Walrasian research programme in macroeconomics. In: DE MARCHI, N.; BLAUG, M. (Ed.). Appraising economic theories: studies in the methodology of research programmes. Aldershot: Edward Elgar, 1991.

BLAUG, M. A methodological appraisal of Marxian economics. Amsterdam: North-Holland, 1980. 
. Kuhn versus Lakatos or paradigm versus research programmes in the history of economics. In: LATSIS, S. Method and appraisal in economics. Cambridge: Cambidge University Press, 1976. Reedição de History of Political Economy, v. 7, n. 4, p. 229-433, 1975.

. Second thoughts on the Keynesian revolution. History of Political Economy, v. 23, n. 2, p. 171-92, 1991.

The methodology of economics: or, how economists explain. Cambridge: Cambridge University Press, 1992.

BROWN, E. K. The neoclassical and post-Keynesian research programmes: the methodological issues. Review of Social Economy, v. 39, n. 2, p. 111-32, 1981.

CHALMERS, A. O que é ciência afinal? São Paulo: Brasiliense, 1981.

DE MARCHI, N. Anomaly and the development of economics: the case of the Leontief paradox. In: LATSIS, S. Method and appraisal in economics. Cambridge: Cambidge University Press, 1976.

DE MARCHI, N. Introduction. In: DE MARCHI, N. (Ed.). Post-Popperian methodology of economics: recovering practice. Boston: Kluwer Academic Publishers, 1992.

DE MARCHI, N. Introduction: rethinking Lakatos. In: DE MARCHI, N.; BLAUG, M. (Ed.). Appraising economic theories: studies in the methodology of research programmes. Aldershot: Edward Elgar, 1991.

EARL, P. E. A behavorial theory of economists' behavior. In: EICHNER, A. S. Why economics is not yet a science. London: Macmillan, 1983.

FAWUNDU, F. Blaug on Kuhn versus Lakatos and the Marginalist revolution. Atlantic Economic Journal, v. 19, n. 1, p. 29-32, 1991.

FISCHER, F. M. The logic of economic discovery. Washington Square, NY: New York University Press, 1986.

FRIEDMAN, M. The methodology of positive economics. In: FRIEDMAN, M. Essays in positive economics. Chicago: The University of Chicago Press, 1953.

GONZALES, W. Lakatos's approach on prediction and novel facts. In: Theoria - Segunda Época, La Coruña, v. 16, n. 3, p. 499-518, 2001.

HANDS, D. W. Falsification, situational analysis and scientific research programs: the Popperian tradition in economic methodology. In: DE MARCHI, N. (Ed.). Post-Popperian methodology of economics: recovering practice. Boston: Kluwer Academic Publishers, 1992.

. Popper and Lakatos in economic methodology. In: MÄKI, U.; GUSTAFSSON, B.; KNUDSEN, C. Rationality, institutions and economic methodology. London: Routledge, 1993.

. Reflection without rules: economic methodology and contemporary science theory. Cambridge: Cambridge University Press, 2001. 
. The problem of excess content: economics, novelty and a long Popperian tale. In: DE $\overline{\mathrm{MARC}}$ I, N.; BLAUG, M. (Ed.). Appraising economic theories: studies in the methodology of research programmes. Aldershot: Edward Elgar, 1991.

HAUSMAN, D. Economics, Philosophy of. 2004. Disponível em: $<$ http://philosophy.wisc.edu/hausman/papers/67.htm>. Acesso em: 22 nov. 2007.

. Kuhn, Lakatos and the character of economics. In: BACKHOUSE, R. New directions in economic methodology. London: Routledge, 1994.

HENDRY, D. Econometrics: alchemy or science? Oxford: Basil Blackwell, 1993.

HOOVER, K. Scientific research programme or tribe? A joint appraisal of Lakatos and the new classical macroeconomics. In: DE MARCHI, N.; BLAUG, M. (Ed.). Appraising economic theories: studies in the methodology of research programmes. Aldershot: Edward Elgar, 1991.

KUHN, T. A estrutura das revoluções científicas. São Paulo: Perspectiva, 1982.

LAKATOS, I. História da ciência e suas reconstruções racionais. Lisboa: Edições 70, 1978.

LATSIS, S. Method and appraisal in economics. Cambridge: Cambridge University Press, 1976.

. Situational determinism in economics. British Journal for the Philosophy of Science, v. 23, n. 3, p. 207-245, 1972.

LEIJONHUFVUD, A. Schools, 'revolutions', and research programmes in economic theory. In: LATSIS, S. Method and appraisal in economics. Cambridge: Cambridge University Press, 1976.

MACHAMER, P. A brief historical introduction to the philosophy of science. Blackwell Sinergy, 2002.

MADDOCK, R. Rational expectations macrotheory: a lakatosian case study in program adjustment. History of Political Economy, v. 16, n. 2, p. 131-52, 1984

MÄKI, U. Method and appraisal in economics, 1976-2006. Talk given on occasion of awarding Dr. Spiro Latsis an Honorary Doctorate ar Witten/Herdecke University, 9 Nov. 2006.

. Methodology of economics: complaints and guidelines. Finnish Economic Papers, v. 3, n. 1, p. 77-84, 1990.

MÄKI, U. Social conditioning of economics. In: DE MARCHI, N. (Ed.). Post-Popperian methodology of economics: recovering practice. Boston: Kluwer Academic Publishers, 1992.

PETRELLA, F. Henry George and the classical scientific research program: the economics of Republican Millenialism. American Journal of Economics and Sociology, v. 47, n. 2, p. 239-56, 1988.

POPPER, K. A lógica da pesquisa científica. São Paulo: Cultrix, 1996.

REDER, M. W. Chicago economics: permanence and change. Journal of Economic Literature, V. 20, n. 1, p. 1-38, 1982. 
RIZZO, M. J. Mises and Lakatos: a reformulation of Austrian methodology. In: KIRZNER, I. M. (Ed.). Method, process, and Austrian economics. Lexington, MA; Toronto: Lexington; D.C. Heath, 1982. p. 53-73.

WEINTRAUB, R. General equilibrium analysis. Cambridge: Cambidge University Press, 1985.

WEINTRAUB, R. Microfoundations: the compability of microeconomics and macroeconomics. New York: Cambridge University Press, 1979.

. The neo-Walrasian program is empirically progressive. In: DE MARCHI, N. (Ed.). The Popperian legacy in economics. Cambridge: Cambidge University Press, 1988. p. 213-27.

WEISS, M. The best way to do economics: moves and countermoves in the history of economic methodology. Durham: Duke University, 2002. (Texto de Discussão). Disponível em: <http://econ.duke.edu/uploads/assets/dje/2002/weiss.pdf>. Acesso em: 11 dez. 2007.

Recebido em: 26/10/2011. Aceito em: 30/05/2014. 